\section{Anticancer Drug Delivery with Transferrin Targeted Polymeric Chitosan Vesicles}

\author{
Christine Dufes, ${ }^{1}$ Jean-Marc Muller, ${ }^{1}$ William Couet, ${ }^{2}$ \\ Jean-Christophe Olivier, ${ }^{2}$ Ijeoma F. Uchegbu, ${ }^{3}$ and \\ Andreas G. Schätzlein ${ }^{4,5}$
}

Received August 4, 2003; accepted September 292003

Purpose. The study reports the initial biological evaluation of targeted polymeric glycol chitosan vesicles as carrier systems for doxorubicin (Dox).

Methods. Transferrin (Tf) was covalently bound to the Dox-loaded palmitoylated glycol chitosan (GCP) vesicles using dimethylsuberimidate (DMSI). For comparison, glucose targeted niosomes were prepared using $N$-palmitoyl glucosamine. Biological properties were studied using confocal microscopy, flow cytometry, and cytotoxicity assays as well as a mouse xenograft model.

Results. Tf vesicles were taken up rapidly with a plateau after $1-2 \mathrm{~h}$ and Dox reached the nucleus after 60-90 min. Uptake was not increased with the use of glucose ligands, but higher uptake and increased cytotoxicity were observed for $\mathrm{Tf}$ targeted as compared to GCP Dox alone. In the drug-resistant A2780AD cells and in A431 cells, the relative increase in activity was significantly higher for the Tf-GCP vesicles than would have been expected from the uptake studies. All vesicle formulations had a superior in vivo safety profile compared to the free drug.

Conclusions. The in vitro advantage of targeted Tf vesicles did not translate into a therapeutic advantage in vivo. All vesicles reduced tumor size on day 2 but were overall less active than the free drug.

KEY WORDS: doxorubicin; glucose niosomes; glycol chitosan; polymeric vesicles; transferrin.

\section{INTRODUCTION}

The clinical use of the broad-spectrum anticancer drug doxorubicin (Dox) can potentially be improved using delivery systems (1).

To improve the specificity of polymeric vesicles, transferrin (Tf) and glucose (Glu) have been coupled to the vesicles (2). The receptors for these ligands are expressed in a range of tumors, but also in some healthy tissues $(3,4)$. Potentially, the combination of active targeting, based on the use of ligands, and passive targeting, based on the accumulation of particulate delivery systems due to the enhanced permeability and retention (5), should provide a tumor-selective

\footnotetext{
${ }^{1}$ Laboratoire de Biologie des Interactions Cellulaires, CNRS UMR 6558, Faculté des Sciences, Poitiers, France.

${ }^{2}$ Laboratoire de Pharmacie Galénique et Biopharmacie, Faculté de Médecine et de Pharmacie, Poitiers, France.

${ }^{3}$ Department of Pharmaceutical Sciences, University of Strathclyde, Strathclyde Institute for Biomedical Sciences, Glasgow, United Kingdom.

${ }^{4}$ Cancer Research UK Department of Medical Oncology, Beatson Laboratories, University of Glasgow, Garscube Estate, Glasgow, G61 1BD, United Kingdom.

5 To whom correspondence should be addressed. (e-mail: A.Schatzlein@beatson.gla.ac.uk)
}

targeting strategy. Even without extravasation, tumor cells that form part of the recently described "mosaic" blood vessels (6) would potentially still be accessible to ligand targeted carriers.

Another motivation for the use of drug carriers as well as targeting ligands is their potential to overcome some acquired mechanisms of drug resistance such as the p-glycoprotein/ MDR1 drug efflux system (7). Additionally, high levels of transferrin expression have been linked with drug resistance (8), offering the possibility of targeting resistant cells with these ligand-bearing particulates.

Furthermore, oral administration of chitosan has been shown to reduce some of the side effects of doxorubicin, in particular the gastrointestinal mucositis after oral administration (9), and it may therefore be possible to improve the safety profile by encapsulation within chitosan-based polymeric vesicles developed in our laboratories (10).

Here we report for the first time the in vivo biological evaluation of doxorubicin formulated in transferrin targeted polymeric vesicles made from palmitoylated glycol chitosan (GCP). We examine whether the previously reported coupling of glucose and transferrin ligands which bind to target receptors overexpressed in some tumors (2) confers a targeting advantage to these systems and whether a modified uptake mechanism could potentially help to overcome drug transport related resistance.

\section{MATERIALS AND METHODS}

$N$-palmitoylglucosamine (2) and palmitoyl glycol chitosan (11) were synthesized and characterized as previously described. Doxorubicin hydrochloride (Dox) was supplied by Alexis Biochemicals (UK). Glycol chitosan had a degree of polymerization of 800 , a degree of acetylation of $33 \mathrm{~mol} \%$, and a degree of palmitoylation of 13 monomer units per 100 monomers. Sorbitan monostearate (Span 60), cholesterol, dimethylsuberimidate dihydrochloride (DMSI), triethanolamine, phosphate-buffered saline (PBS; $\mathrm{pH}=7.4$ ) tablets, iron-saturated human transferrin, Sephadex G50, polyethylene glycol (PEG) 8000, and 3-(4,5-dimethylthiazol-2-yl)-2,5diphenyltetrazolium bromide (MTT) were all purchased from Sigma Aldrich Co. (Poole, UK). Dialysis tubing was obtained from Medicell International (London, UK). Isopropanol and dimethylsulfoxide were purchased from Merck (Nottingham, UK). Cholesteryl poly-24-oxyethylene ether (Solulan C24) was kindly donated by D. F. Anstead (Basildon, UK). A431 cells (CRL-2592) and PC3 cells (CRL-1435) were purchased from the American Type Culture Collection (www.attc.org) and the A2780 cells were originally obtained from Dr. R. F. Ozols (Fox Chase Cancer Center, PA, USA). Selection of the A2780 variant $\mathrm{AD}$ has been described elsewhere (12).

Culture media were obtained from Invitrogen (Pasely, UK). All other tissue culture reagents were obtained from Gibco (Pasely, UK).

\section{Formulation and Characterization}

$N$-palmitoyl glucosamine niosomes (Glu) entrapping doxorubicin were prepared by shaking a mixture of NPG (16 $\mathrm{mg}$ ), Span 60 (65 mg), cholesterol (58 mg), and Solulan C24 (54 $\mathrm{mg})$ in doxorubicin solution $(1.5 \mathrm{mg} / \mathrm{ml}, 2 \mathrm{ml}$, prepared in PBS) at $90^{\circ} \mathrm{C}$ for $1 \mathrm{~h}$, followed by probe sonication for $10 \mathrm{~min}$ (75\% of max). 
Control Span 60 niosomes (Span) were prepared in the same manner from Span 60 (73 mg), cholesterol $(65 \mathrm{mg})$, and Solulan C24 (54 mg) in doxorubicin solution $(1.5 \mathrm{mg} / \mathrm{ml}, 2 \mathrm{ml}$, in PBS).

Palmitoyl glycol chitosan (GCP) vesicles were prepared as previously described (11) by probe sonicating glycol chitosan $(10 \mathrm{mg})$ and cholesterol $(4 \mathrm{mg})$ in doxorubicin solution $(1.5 \mathrm{mg} / \mathrm{ml})$. Tf was linked to the GCP vesicles by crosslinking with DMSI as previously described (2).

For all vesicle suspensions, free drug or ligand (Dox or Tf, respectively) were removed by size exclusion chromatography (Sephadex G50) followed by concentration of the suspensions by dialysis over PEG 8000 .

The amount of conjugated transferrin was determined using the Lowry method as previously described (2).

Doxorubicin loading of control, transferrin-, or glucosebearing vesicles was measured spectrofluorometrically $\left(\lambda_{\mathrm{ex}}=\right.$ $480 \mathrm{~nm}, \lambda_{\mathrm{em}}=560 \mathrm{~nm}$ ) after disruption of vesicles in isopropanol. Vesicle sizing was performed by photon correlation spectroscopy on a Malvern Zetasizer (Malvern Instruments, Malvern, UK). Vesicle preparations were used immediately after preparation and characterization.

\section{Biological Characterization}

\section{Cell Culture}

The human cell lines A431 [epidermoid carcinoma (13)], PC3 [prostate adenocarcinoma (14)], and A2780 (ovarian carcinoma) and its resistant counterpart A2780/AD (15) were grown as monolayers in DMEM (A431 cells) or RPMI 1640 medium supplemented with $10 \%(\mathrm{v} / \mathrm{v})$ fetal bovine serum and $1 \%(\mathrm{v} / \mathrm{v}) \mathrm{L}$-glutamine. Cells were cultured at $37^{\circ} \mathrm{C}$ in a humid atmosphere of $5 \% \mathrm{CO}_{2}(\mathrm{~A} 2780, \mathrm{~A} 2780 / \mathrm{AD})$ or $10 \% \mathrm{CO}_{2}$ (A431, PC3), respectively.

\section{Confocal Microscopy}

Cells were grown on cover slips $\left(\sim 0.6 \times 10^{6}\right.$ cells/ 35-mm dish), washed with PBS ( $\mathrm{pH}=7.4)$, and then transferred to a temperature-controlled holder/chamber $\left(37^{\circ} \mathrm{C}\right)$. Nuclei of the live cells were stained with DAPI $(9 \mu \mathrm{l}, 100 \mu \mathrm{M})$ for 10 min before vesicle suspensions were added $\left(10^{-6} \mathrm{M}\right.$ Dox per well). Cells were examined over time using confocal microscopy $\left(\lambda_{\text {Exc }}=488 \mathrm{~nm}\right.$, Leica, Heidelberg, Germany, TCS SP2) with sensitivity/photo multiplier settings being kept constant. The sum of pixel intensity values (0-254) for each image/time point was used to illustrate the kinetics of Dox uptake (cf. Fig. 1).

\section{Flow Cytometry}

Cells $\left(\sim 1.2 \times 10^{6} / 35-\mathrm{mm}\right.$ dish $)$ were incubated with Dox formulated as vesicles (Tf, GCP, Glu, Span, and free Dox) or as free drug at a final concentration of $3 \times 10^{-7} \mathrm{M}$ Dox per well over $4 \mathrm{~h}$ at $37^{\circ} \mathrm{C}$. Single cell suspensions were prepared, washed $(\mathrm{PBS}, \mathrm{pH}=7.4)$, pelleted $(600 \mathrm{~g})$ twice, and examined on a FACStar flow cytometer (Becton-Dickinson Instruments, Oxford, UK). Twenty thousand cells (gated events) were counted for each sample, and Dox fluorescence was detected with logarithmic settings (FL1; $\lambda_{\mathrm{Em}}=530 \pm 30 \mathrm{~nm}$ ). Cells were counted as positive if their fluorescence (FL1) was higher than that of $95 \%$ of cells from an untreated cell suspension. Each experiment was performed in triplicate and analyzed statistically using one-way analysis of variance (ANOVA), followed by Bonferroni's post-test.

\section{In vitro Cytotoxicity Assay}

Antiproliferative activity of transferrin- or glucosebearing vesicles, PGC vesicles or Span 60 vesicles, all entrapping Dox, were compared with Dox solution in A2780, A2780/AD, A431, and PC3 cell lines. Cells were seeded in quintuplicate (600 cells/96-well), and after 3 days, the medium exchanged with medium containing the formulations at final concentrations of $1 \times 10^{-12} \mathrm{M}$ to $1 \times 10^{-4} \mathrm{M}$. After $4 \mathrm{~h}$, the medium was replaced and the cells further incubated for 3 days. Cytotoxicity was evaluated by measurement of the growth inhibitory concentration for $50 \%$ of the cell population $\left(\mathrm{IC}_{50}\right)$ in a standard MTT [(3-(4,5-dimethylthiazol-2-yl)2,5- diphenyl-tetrazolium bromide blue-indicator dye] -based assay (16). Dose-response curves were fitted to percentage absorbance values to obtain $\mathrm{IC}_{50}$ values (three independent experiments, with $\mathrm{n}=5$ for each concentration level).

\section{In vivo Tumoricidal Activity}

Mice (CD1-nu; weight, $20 \mathrm{~g}$ ) were housed in groups of five (19-23 ${ }^{\circ} \mathrm{C}, 12 \mathrm{~h}$ light-dark cycle) with free access to food (Rat and Mouse Standard Expanded, B and K Universal, Grimston, UK) and water from the mains. Experimental work was carried out in accordance with UK Home Office regulations and approved by the local ethics committee.

Tumors (typical diameter $5 \mathrm{~mm}$ ) were palpable 6 days after implantation of A431 tumor cells ( $10^{6}$ cells per flank). The formulations ( $\mathrm{n}=5$ animals/group) were intravenously administered at a dose equivalent to $10 \mathrm{mg} / \mathrm{kg}$ Dox. Control mice were injected either with Dox solution or received no treatment. The tumor size was measured using callipers and the animals weighed daily. The animals that had received the free Dox solution had to be euthanized after 5 days (i.e., before the planned end of the experiment) because of weight loss. All other animals were killed 10 days after the start of treatment when in some animals the tumor size was already approaching $1 \mathrm{~cm}^{3}$. The tumors were excised and weighed, and tumor size was used as an index for in vivo antitumor activity.

\section{RESULTS}

\section{Vesicle Formulations}

Dox loading into niosomes was $2.9 \times 10^{-3} \pm 0.3 \times 10^{-3} \mathrm{~g} / \mathrm{g}$ of Glu niosome and $2.8 \times 10^{-3} \pm 0.3 \times 10^{-3} \mathrm{~g} / \mathrm{g}$ of control niosomes (Span), respectively, corresponding to $18 \pm 2 \%$ of the initial doxorubicin. Loaded control niosomes had a $z$ average mean diameter of $166 \mathrm{~nm}$ (polydispersity, 0.427) whereas Glu niosomes had a $z$-average mean diameter of 228 $\mathrm{nm}$ (polydispersity, 0.148).

For the GCP vesicles with and without transferrin, the loading was $0.049 \pm 0.005 \mathrm{~g} / \mathrm{g}$, corresponding to $16.3 \pm 1.7 \%$ of the initial doxorubicin. Transferrin was successfully conjugated to GCP vesicles at a level of $0.6 \pm 0.18 \mathrm{~g}$ of transferrin per $g$ polymer $(50 \pm 15 \%$ of the initial transferrin used). The loaded GCP vesicles had a $z$-average mean diameter of 696 $\mathrm{nm}$ (polydispersity, 1) whereas Tf vesicles had a $z$-average size of $889 \mathrm{~nm}$ (polydispersity, 0.0964). 


\section{Biological Characterization}

\section{Confocal Microscopy}

Uptake of free doxorubicin and Tf vesicles was observed using confocal microscopy in live A2780 cells (Fig. 1). Speckled cytoplasmic staining patterns were visible after less than an hour, suggesting some endocytotic uptake (17). Nuclear staining was evident after 60-90 min, suggesting that some Dox-derived fluorescence was either quickly released from endocytotic vesicles or that extracellular doxorubicin leaked from extracellular carriers with some accumulation in a central compartment, possibly the nucleolus. Uptake kinetic was determined in live cells using confocal microscopy (Fig. 1). Untreated cells show a low level of autofluorescence with a narrow distribution of fluorescence intensity. Within only 10 min, an increase of fluorescence intensity, which is not clearly visible yet in the micrographs, can be measured as shift in the mean pixel intensities. Uptake kinetics overall show a sigmoid shape, apparently reaching a maximum after $120 \mathrm{~min}$. Distribution of fluorescence intensities is fairly broad at this point.

\section{Flow Cytometry}

In order to quantify total doxorubicin uptake for different formulations and cell types after $4 \mathrm{~h}$ incubation, we used flow cytometry (Fig. 2). The highest amount of cell-associated fluorescence was found in cells that had been incubated with free doxorubicin (Fig. 3). The mean cell-associated fluorescence for this treatment group was in general more than double that of the best vesicular formulations. Vesicle modification with glucose did not convey any significant improve- ment of uptake in any of the tested cell lines. The brightest fluorescence after vesicular treatment was associated with A431 cells incubated with the Tf vesicles (cf. Fig. 2). Tf vesicle uptake was significantly higher than that of the ligandfree controls in all cell lines. In A431 cells, uptake from Tf vesicles was significantly higher than that of any other vesicle formulation.

\section{In Vitro Cytotoxicity Assay}

Cytotoxicity of doxorubicin formulations was assessed in a panel of cell lines using an MTT-based cell survival assay (Fig. 4) to derive the $\mathrm{IC}_{50}$.

Doxorubicin $\operatorname{IC}_{50}\left(3.55 \times 10^{-8}\right.$ to $\left.7.44 \times 10^{-7}\right)$ is within the range of previously observed values (15). The vesicle formulations were all significantly less toxic in the individual cell lines than the free drug with $\mathrm{IC}_{50}$ ranging from $1.57 \times 10^{-7}$ to $4.83 \times 10^{-6}$. In A431 cells, the Span formulation was found to require 22 times more doxorubicin to kill $50 \%$ of cells than the free drug.

The vesicle formulations tended to have similar levels of cytotoxicity in all cell lines (Fig. 5). The lack of significant differences between targeted (Glu) and nontargeted (Span) niosomes suggests that glucose did not confer a consistent advantage.

While the cytotoxic activity of Tf targeted GCP polymeric vesicles was not significantly different from the other vesicle formulations in the sensitive A2780 cell line, they were about 5-10 times more active than other vesicles in A431 cells. In the resistant A2780/AD cells, they were 3-4 times more effective than GCP vesicles. Interestingly, Tf vesicles were significantly less active than GCP vesicles in PC3 cells.
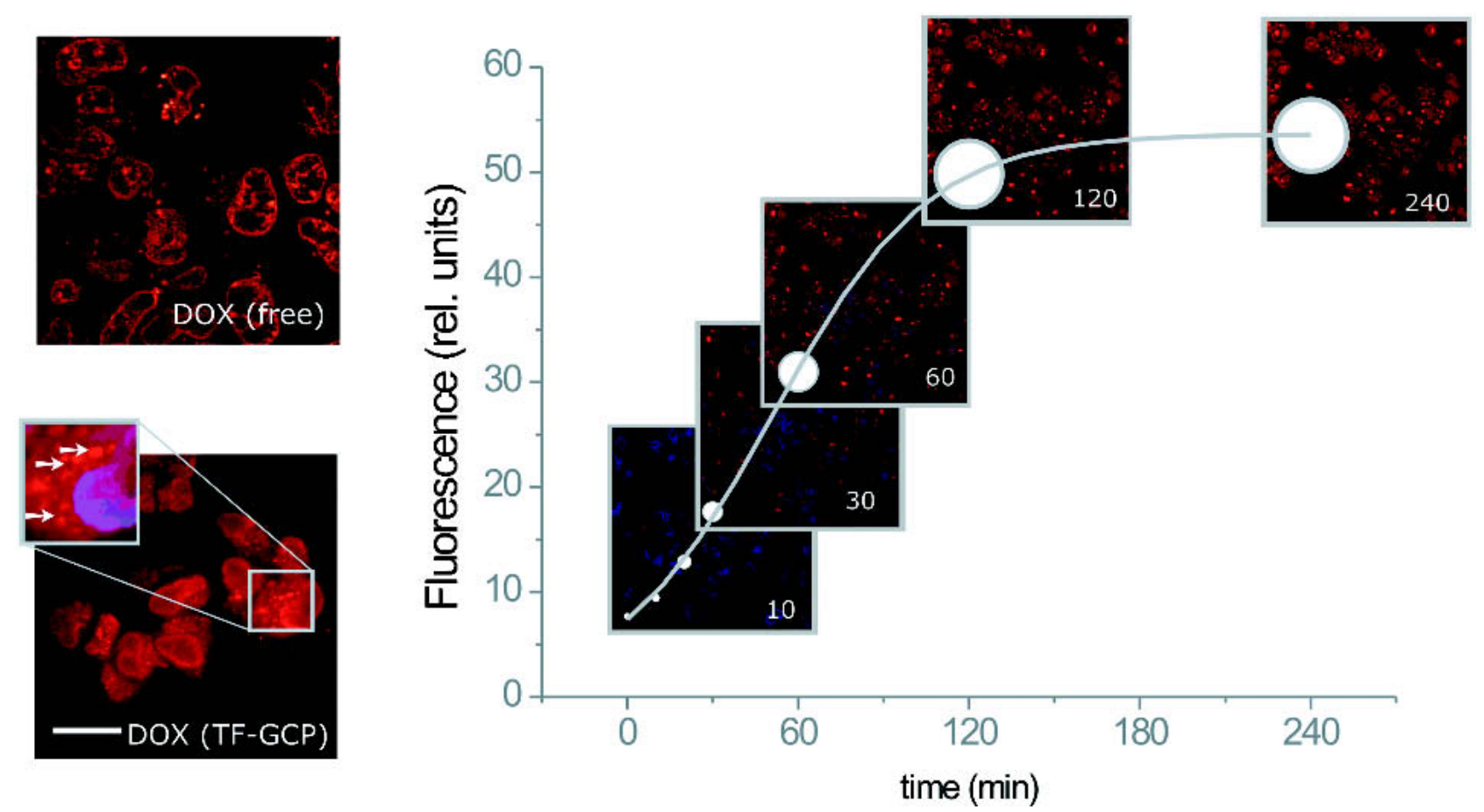

Fig. 1. Confocal microscopy of A2780 cells after incubation with doxorubicin-loaded Tf polymeric vesicles. Left panel shows distribution of free doxorubicin (top) and TF vesicles (bottom) for qualitative comparison of intracellular distribution. The insert in the bottom left panel highlights the speckled appearance of intracellular doxorubicin (arrows). Doxorubicin-derived fluorescence is mainly limited to the nucleus in the case of free doxorubicin (top); scale bar $\sim 50 \mu \mathrm{m}$. Right panel: The graph visualizes the kinetics of Dox uptake into A2780 cells when incubated with doxorubicin-loaded Tf polymeric vesicles. Circles represent range of distribution of pixel intensities of the corresponding confocal images. 


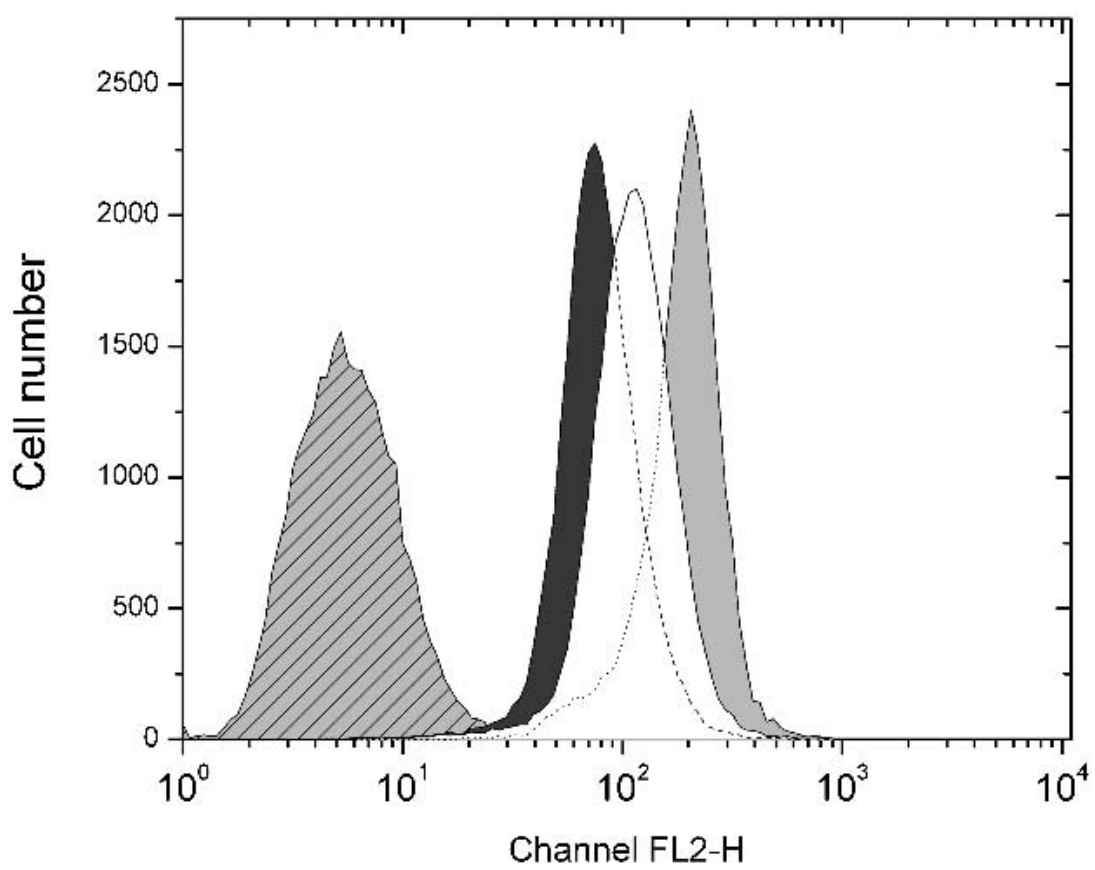

Fig. 2. Flow cytometric measurement of doxorubicin uptake into A431 cells. Untreated cells (hashed, gray) served as negative control while free doxorubicin solution was used as positive control (gray). Doxorubicin uptake from GCP vesicles (black) and transferrin targeted GCP vesicles (white) were compared after $4 \mathrm{~h}$ incubation at $37^{\circ} \mathrm{C}$.

\section{In Vivo Tumoricidal Activity and Toxicity}

In vivo activity was tested in an A431 xenograft model with established tumors using a single dose of the formulations (Fig. 6). Starting tumor size of treated groups was not statistically significantly different from the control group. While the doxorubicin dose was efficient in suppressing further tumor growth, we did not observe a reduction of tumor size. More importantly, treatment of the animals with free

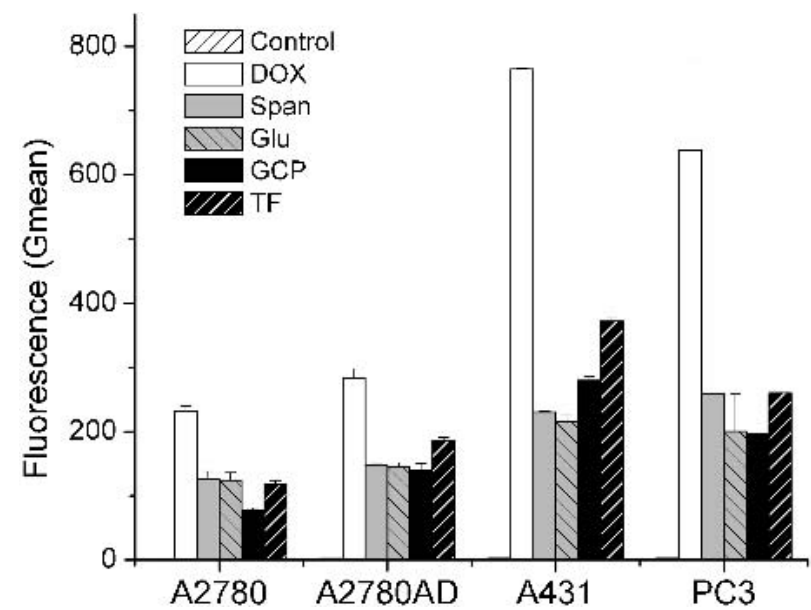

Fig. 3. Flow cytometric analysis $(n=3)$ of the uptake of Dox solution (white bar), Dox-loaded Span 60 vesicles without glucose (light gray), glucose-bearing vesicles (gray, black hash), GCP vesicles without transferrin (dark gray), transferrin-bearing vesicles (hashed white) by A2780, A2780/AD, A431, and PC3 cells. Untreated cells (represented by the first bar in each group; white, black hash) are not visible at this scale. Cells were counted as Dox positive when their fluorescence was higher than that of $95 \%$ of cells from an untreated cell suspension. doxorubicin at this dose level induced significant signs of toxicity; that is, a pronounced weight loss and all animals in this group had to be euthanized on day 5 (Fig. 7).

The response of the vesicular formulations in the A431 xenograft model were not significantly different, and treated groups could not be distinguished from the control group, except during the first $24 \mathrm{~h}$ after injection when targeted or control vesicles were found to be significantly more active than Dox in solution, with a reduction in size between 1 day and 2 days after injection being significant for all the vesicle groups (Tf, p < 0.05; GCP, p < 0.01; Span, p < 0.001; Glu, $\mathrm{p}<0.001)$

In all vesicle groups as well as the control group, all of the animals appeared lively throughout the study, and no weight loss was detected. There were no signs of decreased activity, which would indicate general toxicity. As a result, vesicles are considered to be safe at the dosing schedule used.

\section{DISCUSSION}

Transferrin receptors are found on the surface of most proliferating cells and, in elevated numbers, on erythroblasts and on many tumors $\left(1.5 \times 10^{5}\right.$ to $10^{6} /$ cell) (3) where they have been linked to drug resistance (8). The facilitative glucose transporter GLUT-1 has been correlated to the transition to malignancy and response to chemotherapy $(4,18)$. We have recently reported targeting of doxorubicin-loaded polymeric vesicles from palmitoylated glycol chitosan in vitro using the ligands transferrin and glucosamine, which bind to these receptors (2). Here we report the evaluation of these systems in vivo as potential delivery systems to solid tumors, comparing them with well-characterized niosome formulations.

Doxorubicin was chosen as a model drug with potential 


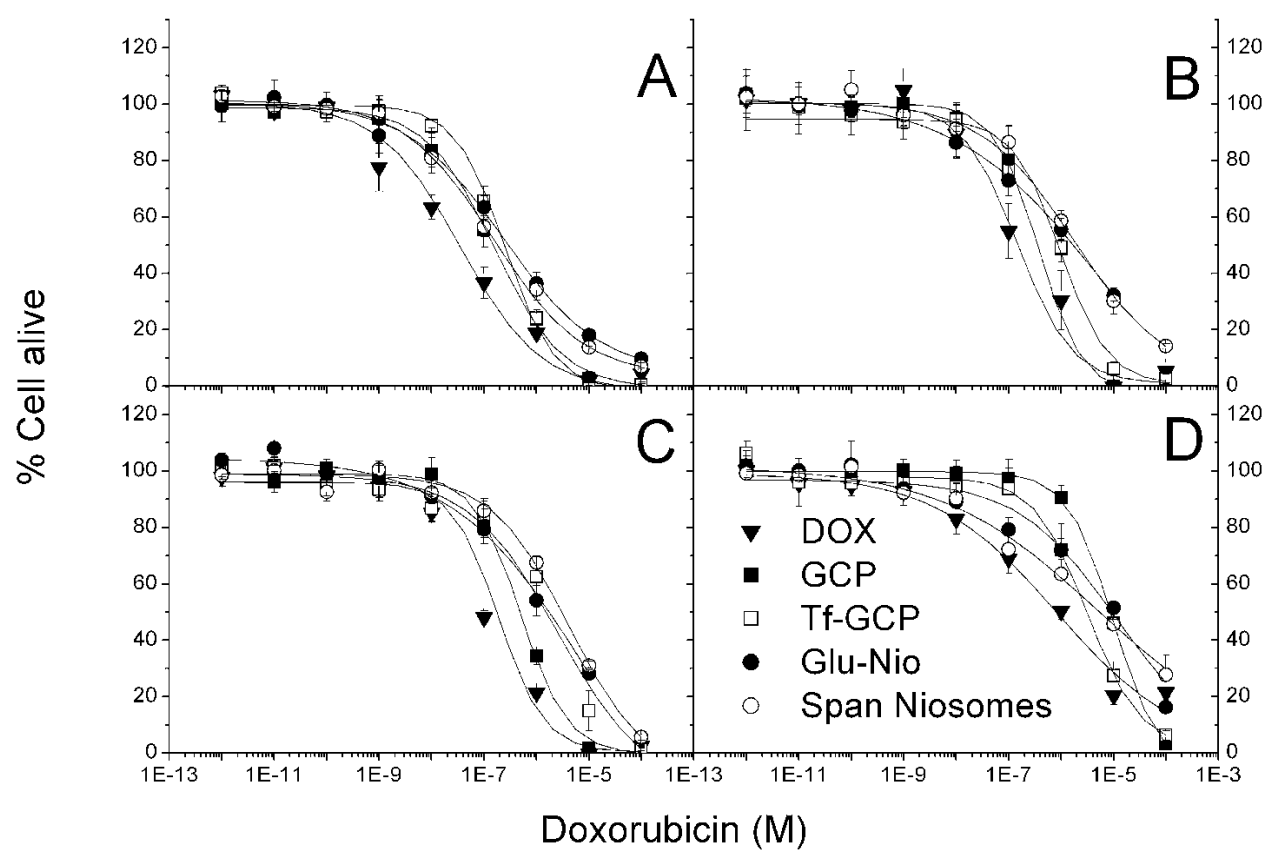

Fig. 4. Cytotoxicity of Dox delivered as free drug (Dox), loaded in Span 60 vesicles without glucose (Span), glucose-bearing vesicles (Glu), GCP vesicles without transferrin (GCP), transferrin-bearing vesicles (Tf), against (A) A2780, (B) A2780/AD, (C) A431, and (D) PC3 cells $(n=15)$. Error $=$ SE.

therapeutic relevance because of the ease of detection (microscopy, FACS, HPLC). Doxorubicin has been in clinical use against a wide range of human cancers for decades. Nevertheless, a number of issues critical to the therapeutic success and safety of the drug, such as cardiotoxicity, drug resistance, and specificity remain unresolved.

Tumor growth beyond a few millimeters requires recruitment of additional blood vessels, which exhibit a large number of cellular holes and gaps (19). The resulting irregularity in the fluid flow has been used with good effect to target macromolecular and particulate drug carriers to tumors (20).

For a majority of tumors, the cut-off size for extravasation has been found to be below $400 \mathrm{~nm}$ (21-23), suggesting that larger vesicles may be at a disadvantage. However, a larger variation of cut-off size [the cut-off ranging from between $200 \mathrm{~nm}$ and $1.2 \mu \mathrm{m}$ or even up to $2 \mu \mathrm{m}$ (19)] has

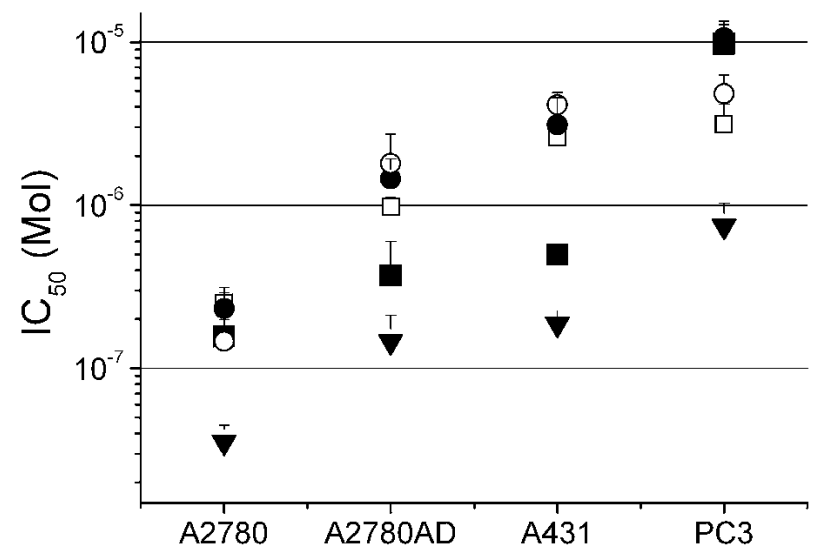

Fig. 5. Cytotoxicity of free Dox $(\mathbf{\nabla})$ and of doxorubicin formulated as Span niosomes $(\bigcirc)$, Glu niosomes $(\bullet)$, GCP vesicles $(\square)$, or Tf vesicles (ם) in A2780, A2780AD, A431, and PC3 cells expressed as $\mathrm{IC}_{50}$ values. $\mathrm{n}=15$. recently been described. The pore size showed significant heterogeneity in the tumor and a strong dependence on tumor type and microenvironment (24).

Furthermore, it has been demonstrated that a significant proportion of the tumor vasculature in colon carcinoma xenografts was made up of tumor cells themselves (6). In these "mosaic" type vessels, a targeted carrier would potentially have direct access to the receptor-expressing tumor cells without the need for extravasation.

Both ligands, glucose and transferrin, were chosen because they could potentially modulate uptake in a broader range of tumors. Therefore the combination of two targeting mechanisms (active targeting using the TF ligand and passive

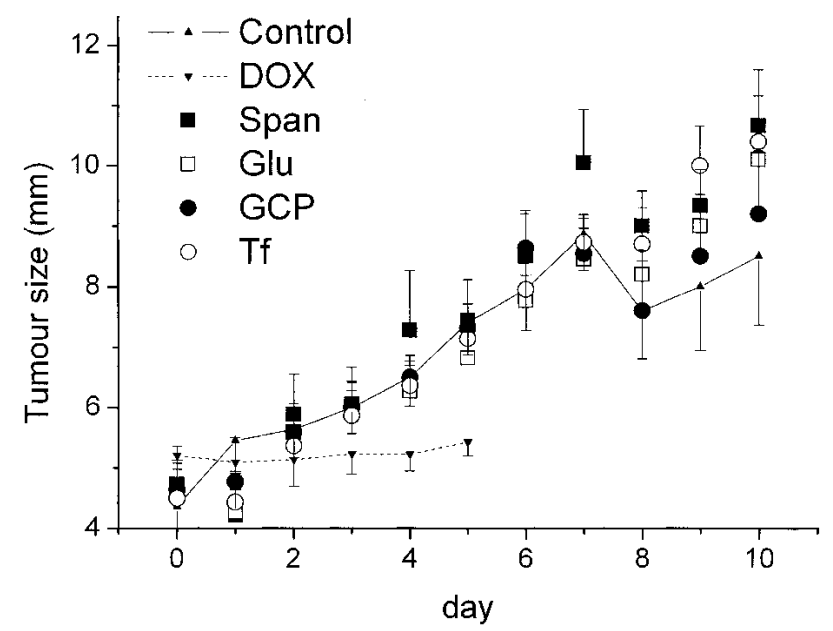

Fig. 6. Tumoricidal activity of intravenously injected Dox solution $(\boldsymbol{\nabla})$, Span niosomes $(\boldsymbol{\square})$, Glu niosomes $(\square)$, GCP vesicles $(\bullet)$, and Tf vesicles $(\bigcirc)$ against an A431 tumor subcutaneously implanted in nude mice $(\mathrm{n}=5)$. Untreated animals served as controls $(\mathbf{\Delta})$. Error $=\mathrm{SE}$ 


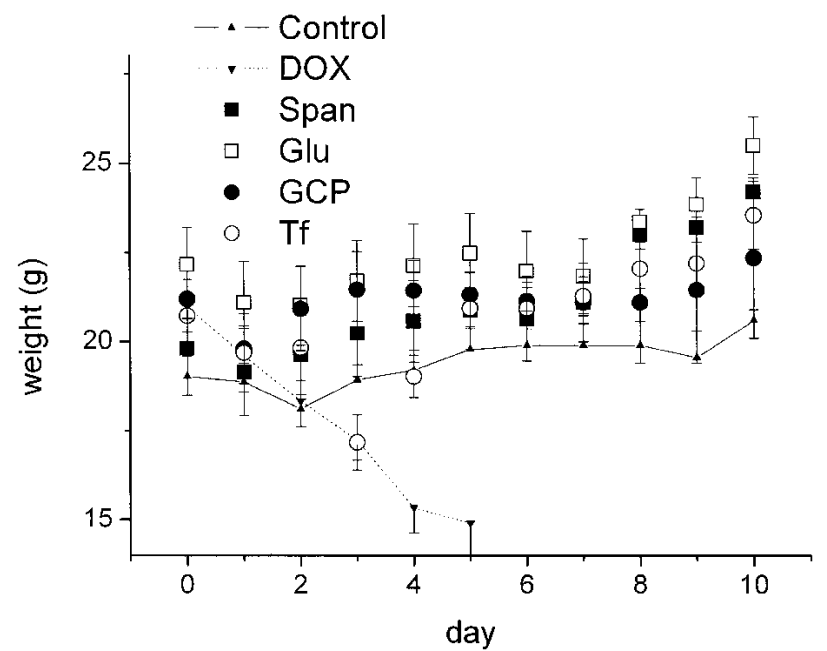

Fig. 7. Daily weights of A431 tumor-bearing nude mice intravenously injected with Dox solution $(\boldsymbol{\nabla})$, Span niosomes $(\boldsymbol{\square})$, Glu niosomes $(\square)$, GCP vesicles $(\mathbf{O})$, and Tf GCP vesicles $(\bigcirc)$. Untreated animals served as controls $(\boldsymbol{A}) . \mathrm{n}=5$.

targeting using the EPR effect) could potentially provide a tumor-selective targeting strategy.

The cellular distribution over time seen with the vesicles is distinctly different from the patterns observed with the free drug (Fig. 1), and within 20-30 min shows a pattern typically observed with endocytotic uptake of particulate carriers (17). But already after 60-90 min, there is also evidence of nuclear accumulation of doxorubicin (Fig. 1).

Between 60-120 min, the rate of uptake appears to slow down, and the total amount taken up approaches a maximum (Fig. 1). This effect was not observed with free doxorubicin (data not shown), suggesting that self-quenching of Dox fluorescence did not play a major role in this observation. The effect may be linked to a depletion of transferrin receptors on the cell surface possibly linked to the intracellular retention of the receptor after multivalent binding events (25).

Free doxorubicin enters the cell by diffusion, leading to significantly higher drug levels than found with the vesicular formulations (Fig. 3) that are taken up via endocytosis, a comparatively slower but potentially highly specific process. Despite the ability of the glucose targeted vesicles to bind concavalin A (2), there was no modulation of cellular uptake of the vesicles compared to the unmodified control niosomes. The Tf targeted vesicles on the other hand increased the uptake in all cell lines with a relative improvement factor $1.37 \pm$ 0.1 when compared to nontargeted GCP vesicles.

We then examined whether the various levels of cellular Dox uptake are linked to similar differences in the level of cytotoxicity of the various formulations. While glucose targeted niosomes did not enhance doxorubicin cytotoxicity when compared to plain niosomes in any of the cell lines, the addition of transferrin to the surface of the vesicles increased cytotoxic activity in some of the cell lines (Fig. 5). The uptake assay clearly showed moderately increased uptake for the transferrin-targeted vesicles by factor of 1.37 , which for the A2780 cell line was consistent with the improved cytotoxicity observed (factor 1.6). However, in the doxorubicin-resistant A2780/AD cell line, the activity improvement (factor 2.6) was found to be over and above the uptake advantage. This was even more evident for the A431 cell line where the transferrin conferred a 5.2-fold higher activity. The A431 cells are known to express high levels of transferrin receptor (TfR), and, in the A2780/AD cell line, improvement may also be linked with a relatively higher Tf receptor activity (8). Surprisingly, in the PC3 cell line, the improved uptake is not reflected in the level of activity; in fact, the nontargeted formulation is 3 times more toxic than the targeted formulation in this cell line. The reason for this is unclear.

As expected, the activity of vesicular formulations was lower than that of the free drug. However, there were marked differences ranging over almost one order of magnitude in the same cell line (factor 2.67 for Tf and 22.04 for Span). However, when the formulations were tested in vivo in A431 xenograft models, the results did not bear out the initial promise. None of the vesicular formulations was able to delay significantly tumor growth after a single dose. The free drug did not lead to tumor shrinkage but stopped further increase in tumor size before the animals had to be killed because of the severe weight loss.

Although the Dox dose of $10 \mathrm{mg} / \mathrm{kg}$ was not sufficient to induce cures in any of the mice, it halted further tumor growth. On the other hand, the dose was linked to a significant toxicity in all the mice in this control group (Fig. 7) despite having been used previously in another mouse strain (26-28). As the activity of the free Dox in vitro was only 2.5 times higher than that of the Tf vesicles in vitro, it is somewhat surprising that this formulation did not show any clear signs of therapeutic effect, suggesting problems linked to the delivery in vivo.

Studies using doxorubicin Span 60 niosomes indicated an improvement in tumoricidal activity with a similar single dose on MAC 15A tumor and CH1 Dox ${ }^{\mathrm{R}}$ tumor $(27,28)$. One potential problem of the formulations used could lie in the relatively large size of the vesicles (around $890 \mathrm{~nm}$ ). Transvascular transport of particulate delivery systems depends on gaps in the tumor blood vessels, which in many mouse tumor models range from $0.2 \mu \mathrm{m}-1.2 \mu \mathrm{m}$ (24). While still within the range of cut-off sizes, the vesicles used in this study were relatively large $(890 \mathrm{~nm})$, and their extravasation in the A431 tumor could have been impaired. The size increase from 690 $\mathrm{nm}$ to $890 \mathrm{~nm}$ induced by Tf coupling and the purification procedure may be unavoidable, but we have recently demonstrated that the starting size of the GCP polymeric vesicles can be modulated by control of the molecular weight of the starting material (29), thus offering a strategy of adjusting the delivery system to the vascular properties of a given tumor. Alternatively, a pharmacological augmentation or transport may be possible (30).

In summary vesicles retain doxorubicin and altered the uptake into cells (Fig. 1) in a fashion consistent with endosomal uptake of particulate carriers. The transferrinconjugated vesicles showed a statistically significant uptake advantage when compared to the nontargeted vesicles (cf. Fig. 3). The level of association after $4 \mathrm{~h}$ is consistently increased by more than $30 \%$ in contrast to the glucose targeted niosomes, where levels are unchanged or slightly decreased. While no significant difference in cytotoxicity was observed between the vesicular formulations in the highly doxorubicin sensitive cell line A2780, the Tf targeted vesicles demonstrated a significant improvement of activity in the A2780- 
derived resistant cell line and the resistant A431 cell line (265\% and $521 \%$, respectively).

It is encouraging that in preliminary in vivo studies, use of a single dose of systemically administered vesicles has not only been shown to avoid the toxicity of the free drug, but also to inhibit the growth of A431 cancer cells, if only at the beginning of the treatment. There may be scope to improve on the in vivo activity of these extremely safe delivery systems by using a higher dose, reducing the size of the vesicles (29), and improving the targeting/coupling. Thus, it may be possible to improve specificity and efficacy of these delivery systems by combination of semiselective passive and active targeting strategies.

\section{ACKNOWLEDGMENTS}

C. D. would like to thank the Comité Départemental de la Vienne of the Ligue contre le Cancer Association (France) for its financial support. J. C. O. is a member of the emerging Anti-Infectious Drugs and Blood Brain Barrier team of the University of Poitiers.

\section{REFERENCES}

1. M. Winterhalter, P. M. Frederik, J. J. Vallner, and D. D. Lasic. Stealth(R) liposomes: from theory to product. Adv. Drug Deliv. Rev. 24:165-177 (1997).

2. C. Dufes, A. G. Schätzlein, L. Tetley, A. I. Gray, D. G. Watson, J. C. Olivier, W. Couet, and I. F. Uchegbu. Niosomes and polymeric chitosan based vesicles bearing transferrin and glucose ligands for drug targeting. Pharm. Res. 17:1250-1258 (2000).

3. J. Goding. CD71, NCBI Proteins on the Web. Available: http:// www.ncbi.nlm.nih.gov/PROW/guide/1445562251_g.htm (1999).

4. J. D. Chandler, E. D. Williams, J. L. Slavin, J. D. Best, and S. Rogers. Expression and localization of GLUT1 and GLUT12 in prostate carcinoma. Cancer 97:2035-2042 (2003).

5. H. Maeda. The tumor blood vessel as an ideal target for macromolecular anticancer agents. J Controlled Release 19:315-324 (1992)

6. Y. S. Chang, E. di Tomaso, D. M. McDonald, R. Jones, R. K. Jain, and L. L. Munn. Mosaic blood vessels in tumors: frequency of cancer cells in contact with flowing blood. Proc. Natl. Acad. Sci. U. S. A. 97:14608-14613 (2000).

7. A. K. Larsen, A. E. Escargueil, and A. Skladanowski. Resistance mechanisms associated with altered intracellular distribution of anticancer agents. Pharmacol. Ther. 85:217-229 (2000).

8. K. Barabas and W. P. Faulk. Transferrin receptors associate with drug resistance in cancer cells. Biochem. Biophys. Res. Commun. 197:702-708 (1993).

9. Y. Kimura, N. Sawai, and H. Okuda. Antitumour activity and adverse reactions of combined treatment with chitosan and doxorubicin in tumor-bearing mice. J. Pharm. Pharmacol. 53:13731378 (2001).

10. I. F. Uchegbu, L. Tetley, and A. G. Schätzlein. Polymeric palmitoyl glycol chitosan (GP) vesicles - a new drug delivery system. $J$. Pharm. Pharmacol. 49:S27 (1997).

11. I. F. Uchegbu, A. G. Schätzlein, L. Tetley, A. I. Gray, J. Sludden, S. Siddique, and E. Mosha. Polymeric chitosan-based vesicles for drug delivery. J. Pharm. Pharmacol. 50:453-458 (1998).

12. D. A. Anthoney, A. J. McIlwrath, W. M. Gallagher, A. R. Edlin, and R. Brown. Microsatellite instability, apoptosis, and loss of p53 function in drug-resistant tumor cells. Cancer Res. 56:13741381 (1996)

13. D. J. Giard, S. A. Aaronson, G. J. Todaro, P. Arnstein, J. H.
Kersey, H. Dosik, and W. P. Parks. In vitro cultivation of human tumors: establishment of cell lines derived from a series of solid tumors. J. Natl. Cancer Inst. 51:1417-1423 (1973).

14. M. C. Rossi and B. R. Zetter. Selective stimulation of prostatic carcinoma cell proliferation by transferrin. Proc. Natl. Acad. Sci. U. S. A. 89:6197-6201 (1992).

15. T. Minko, P. Kopeckova, V. Pozharov, and J. Kopecek. HPMA copolymer bound adriamycin overcomes MDR1 gene encoded resistance in a human ovarian carcinoma cell line. $J$ Controlled Release 54:223-233 (1998).

16. J. A. Plumb, R. Milroy, and S. B. Kaye. Effects of the $\mathrm{pH}$ dependence of 3-(4,5-dimethylthiazol-2-yl)-2,5-diphenyltetrazolium bromide-formazan absorption on chemosensitivity determined by a novel tetrazolium-based assay. Cancer Res. 49: 4435-4440 (1989).

17. M. Hao and F. R. Maxfield. Characterization of rapid membrane internalization and recycling. J. Biol. Chem. 275:15279-15286 (2000).

18. G. Cantuaria, A. Fagotti, G. Ferrandina, A. Magalhaes, M. Nadji, R. Angioli, M. Penalver, S. Mancuso, and G. Scambia. GLUT-1 expression in ovarian carcinoma: association with survival and response to chemotherapy. Cancer 92:1144-1150 (2001).

19. H. Hashizume, P. Baluk, S. Morikawa, J. W. McLean, G. Thurston, S. Roberge, R. K. Jain, and D. M. McDonald. Openings between defective endothelial cells explain tumor vessel leakiness. Am. J. Pathol. 156:1363-1380 (2000)

20. R. Duncan. The dawning era of polymer therapeutics. Nat. Rev. Drug Discov. 2:347-360 (2003).

21. G. Kong, R. D. Braun, and M. W. Dewhirst. Hyperthermia enables tumor-specific nanoparticle delivery: effect of particle size. Cancer Res. 60:4440-4445 (2000).

22. O. Ishida, K. Maruyama, K. Sasaki, and M. Iwatsuru. Sizedependent extravasation and interstitial localization of polyethyleneglycol liposomes in solid tumor-bearing mice. Int. J. Pharm. 190:49-56 (1999).

23. F. Yuan, M. Dellian, D. Fukumura, M. Leunig, D. A. Berk, V. P. Torchilin, and R. K. Jain. Vascular permeability in a human tumor xenograft: molecular size dependence and cutoff size. Cancer Res. 55:3752-3756 (1995).

24. S. K. Hobbs, W. L. Monsky, F. Yuan, W. G. Roberts, L. Griffith, V. P. Torchilin, and R. K. Jain. Regulation of transport pathways in tumor vessels: role of tumor type and microenvironment. Proc. Natl. Acad. Sci. U. S. A. 95:4607-4612 (1998).

25. E. W. Marsh, P. L. Leopold, N. L. Jones, and F. R. Maxfield. Oligomerized transferrin receptors are selectively retained by a lumenal sorting signal in a long-lived endocytic recycling compartment. J. Cell Biol. 129:1509-1522 (1995).

26. P. M. Loadman, M. C. Bibby, J. A. Double, W. M. Al-Shakhaa, and R. Duncan. Pharmacokinetics of PK1 and doxorubicin in experimental colon tumor models with differing responses to PK1. Clin. Cancer Res. 5:3682-3688 (1999).

27. I. F. Uchegbu, J. A. Double, J. A. Turton, and A. T. Florence. Distribution, metabolism and tumoricidal activity of doxorubicin administered in sorbitan monostearate (Span 60) niosomes in the mouse. Pharm. Res. 12:1019-1024 (1995).

28. I. F. Uchegbu, J. A. Double, L. R. Kelland, J. A. Turton, and A. T. Florence. The activity of doxorubicin niosomes against an ovarian cancer cell line and three in vivo mouse tumor models. J. Drug Target. 3:399-409 (1996).

29. W. Wang, L. Tetley, A. M. McConaghy, and I. F. Uchegbu. Controls on polymer molecular weight may be used to control the size of palmitoyl glycol chitosan polymeric vesicles. Langmuir 17:631636 (2001).

30. W. L. Monsky, D. Fukumura, T. Gohongi, M. Ancukiewcz, H. A. Weich, V. P. Torchilin, F. Yuan, and R. K. Jain. Augmentation of transvascular transport of macromolecules and nanoparticles in tumors using vascular endothelial growth factor. Cancer Res. 59: 4129-4135 (1999). 